\title{
Bacterial Infection and Predictors of Mortality in Patients with Autoimmune Liver Disease-Associated Acute-On-Chronic Liver Failure
}

\author{
Xuan Zhang, ${ }^{1}$ Ping Chen, ${ }^{2}$ Hainv Gao, ${ }^{2}$ Shaorui Hao, ${ }^{1}$ Meifang Yang, ${ }^{1}$ Hong Zhao, ${ }^{1}$ \\ Jianhua $\mathrm{Hu},{ }^{1}$ Weihang $\mathrm{Ma},{ }^{1}$ and Lanjuan $\mathrm{Li} \mathbb{D D}^{1}$ \\ ${ }^{1}$ State Key Laboratory for Diagnosis and Treatment of Infectious Diseases, Collaborative Innovation Center for Diagnosis and \\ Treatment of Infectious Diseases, The First Affiliated Hospital, College of Medicine, Zhejiang University, Hangzhou 310003, China \\ ${ }^{2}$ Infectious disease department, Shulan (Hangzhou) Hospital, Hangzhou, China
}

Correspondence should be addressed to Lanjuan Li; ljli@zju.edu.cn

Received 28 September 2017; Revised 13 December 2017; Accepted 11 January 2018; Published 28 January 2018

Academic Editor: Yu-Chen Fan

Copyright (C) 2018 Xuan Zhang et al. This is an open access article distributed under the Creative Commons Attribution License, which permits unrestricted use, distribution, and reproduction in any medium, provided the original work is properly cited.

Objective. To date, few studies are available on autoimmune liver disease-associated acute-on-chronic liver failure (ACLF). The aim of this study is to investigate bacterial infection and predictors of mortality in these patients. Methods. We retrospectively studied patients with autoimmune liver disease from August 2012 to August 2017. Clinical data of the patients were retrieved for analysis. Results. There were 53 ACLF patients and 53 patients without ACLF in this study. The ACLF group had a higher prevalence of complications $(P<0.05)$. The 28 -day and 90 -day mortality rates were also obviously high in patients with ACLF (38.3\% and $74.5 \%$, resp. $)(P<0.05)$. No predictor was significantly associated with 28 -day and 90 -day transplant-free mortality. In 53ACLF patients, 40 (75.5\%) patients showed bacterial infection. ACLF patients with bacterial infection showed high Child-Pugh score, MELD score, CLIF-SOFA score, 28-day mortality, and 90-day mortality $(P>0.05)$. Moreover, C-reactive protein $(\mathrm{CRP})$ using 12.15 $\mathrm{mg} / \mathrm{L}$ cut-off value proved to be more accurate than procalcitonin in identifying patients with infection. Conclusions. Autoimmune liver disease-associated ACLF showed more complications and high mortality. Bacterial infection patients displayed a more severe condition than those without infection. Elevated CRP is an accurate marker for diagnosing bacterial infection in autoimmune liver disease-associated ACLF patients.

\section{Introduction}

In recent years, acute-on-chronic liver failure (ACLF) as a specific clinical form of liver failure has attracted increasing attention. In fact, ACLF is considered a syndrome that occurs on the background of chronic liver disease, and previously diagnosed cirrhosis is not required, which is characterized by acute hepatic decompensation resulting in liver failure (jaundice and prolongation of the international normalized ratio [INR]) and one or more extrahepatic organ failures that are associated with increased mortality within a period of 28 days and up to three months from onset $[1,2]$. Moreover, ACLF can rapidly progress, requiring an urgent need for assessment and referral for liver transplantation [3].
Therefore, recognition and intervention of the predictors of mortality in ACLF patients can prevent or reverse the process and improve the survival rate.

Several patients with ACLF have been recently reported [3-7]. Moreau et al. found that bacterial infection is the trigger of $33 \%$ ACLF and is the most commonly identifiable trigger of this syndrome [4]. Some studies considered hepatic encephalopathy, low-serum sodium, and high INR as predictors of poor outcome in ACLF patients $[3,5]$. However, few studies about autoimmune liver disease-induced ACLF patients are available to date. Except for the known predictors, possible risk factors have also received less attention. Moreover, bacterial infection in the population is not largely known. The aim of this study is, therefore, to collect data 
about autoimmune liver disease-associated ACLF patients to investigate bacterial infection and predictors of mortality to reduce the mortality in this population.

\section{Materials and Methods}

2.1. Study Population and Data Collection. We retrospectively analyzed the data of all patients admitted at the infectious disease wards of the First Affiliated Hospital, College of Medicine, Zhejiang University, China, and diagnosed with autoimmune liver disease-associated ACLF from August 2012 to August 2017. Patients with autoimmune liver disease who satisfied the 13th Asia-Pacific Congress of Clinical Microbiology and Infection (APCCMI) Consensus Guidelines for diagnosis and treatment of liver failure [8], but not diagnostic criteria of the European Association for the Study of the Liver (EASL) [4], were as control group (i.e., no ACLF group). All of the patients in our study had oral care after they admitted to hospital. Patients showing the following were excluded: coinfections with other viruses, including hepatitis A virus, hepatitis $B$ virus, hepatitis $C$ virus, hepatitis $D$ virus, hepatitis $\mathrm{E}$ virus, and human immunodeficiency virus; concomitant liver diseases, such as Wilson's disease; coexisting liver cancer or extrahepatic malignancy; usage of hepatotoxic drugs. We used the composite of death or liver transplantation as our endpoint.

The research protocol was reviewed and approved by the Ethics Committee of the First Affiliated Hospital of Zhejiang University. The need for consent was waived because the study was retrospective and data were analyzed anonymously.

The following data were collected from hospital information system and medical documents: age, gender, diabetes, coexisting other autoimmune diseases, steroid exposure, laboratory findings, symptoms, the presence of bacterial infection at admission or during hospitalization, and mortality at 28 and 90 days. The chronic liver failure-sequential organ failure assessment (CLIF-SOFA) score, Child-Pugh score, and model for end-stage liver disease (MELD) score were calculated from the collected data.

2.2. Definitions. Diagnostic criteria of primary biliary cholangitis (PBC), autoimmune hepatitis ( $\mathrm{AIH})$, and primary sclerosing cholangitis (PSC) were defined by American Association for the Study of Liver Diseases (AASLD) [9-11]. Diagnosis of AIH-PBC overlap syndrome referenced the standard which was proposed by Chazouillères et al. in 1998 [12].

Diagnostic criteria and grades of ACLF were defined according to EASL definition [4], as follows.

ACLF Grade 1. This group includes 3 subgroups: (1) patients with single kidney failure, (2) patients with single failure of the liver, coagulation, circulation, or respiration who had a serum creatinine level ranging from 1.5 to $1.9 \mathrm{mg} / \mathrm{dL}$ and/or mild to moderate hepatic encephalopathy, and (3) patients with single cerebral failure who had a serum creatinine level ranging from 1.5 and $1.9 \mathrm{mg} / \mathrm{dL}$.
ACLF Grade 2. This group includes the patients with 2 organ failures.

ACLF Grade 3. This group includes the patients with 3 organ failures or more.

The patients as control group satisfied the following criteria specified by the 13th APCCMI Consensus Guidelines for diagnosis and treatment of liver failure [8] as follows.

Patients with chronic liver diseases have acute or subacute deterioration of liver function. ACLF usually exhibits the following symptoms: (a) fatigue with gastrointestinal tract symptoms; (b) rapidly deepening jaundice, with total bilirubin 10 times higher than the upper limit of normal or a daily increase $\geq 17.1 \mu \mathrm{mol} / \mathrm{L}$; (c) hemorrhagic tendency with INR $\geq 1.5$ or prothrombin activity $\leq 40 \%$ and other causes which have been excluded; (d) progressive reduction in liver size; and (e) hepatic encephalopathy occurrence.

Bacterial infection in parts of the body was defined as follows [13]. Bacterial pneumonia was defined as the association of clinical and radiological signs of lung infection observed in chest radiographs. Spontaneous bacterial peritonitis was diagnosed when ascites culture was positive or polymorphonuclear count was no less than 250 cells $/ \mu \mathrm{L}$ in ascites, excluding other inflammations such as pancreatitis, peritoneal carcinosis, tuberculosis, and bloody ascites. Urinary tract infection was diagnosed using bacterial culture positive or urine leukocyte count $>15$ cells/high power field and $>10^{6}$ bacteria $/ \mu \mathrm{L}$. Fever and cellulitis associated with leukocytosis were used to diagnose skin and soft tissue infection. Septicemia was defined as clinical signs of infection and two consecutive blood cultures yielding the same organism, when a blood culture yielding an organism was considered as bacteremia. Patients considered for bacterial infections but without positive culture or evidence of organ involvement were considered as undetermined infection.

2.3. Statistical Analyses. Statistical analysis was performed using SPSS version 18.0 (SPSS, Chicago, IL, USA). Categorical variables were expressed in percentages and frequencies. Continuous variables were expressed as means and standard deviation. Continuous variables were analyzed with independent-sample $t$-test when they in line with the normal distribution otherwise Mann-Whitney $U$ test was used. The chi-square test was performed to analyze categorical variables. The 90-day mortality prediction was carried out with univariate and multivariate logistic regression. $P$ value $<0.05$ was considered statistically significant.

\section{Result}

3.1. Characteristics of the Study Cohort. During the study period, 53 patients with autoimmune liver disease-associated ACLF who were admitted to the infectious disease ward of our hospital were included in this study. Fifty-three patients were included as control group. The clinical features and laboratory results of the ACLF patients and no ACLF patients are shown in Table 1. From these 53 ACLF patients, 30 patients (56.6\%) showed PBC, 21 (39.6\%) displayed AIH, 1 (1.9\%) had PSC, and one (1.9\%) patients had AIH-PBC overlap 


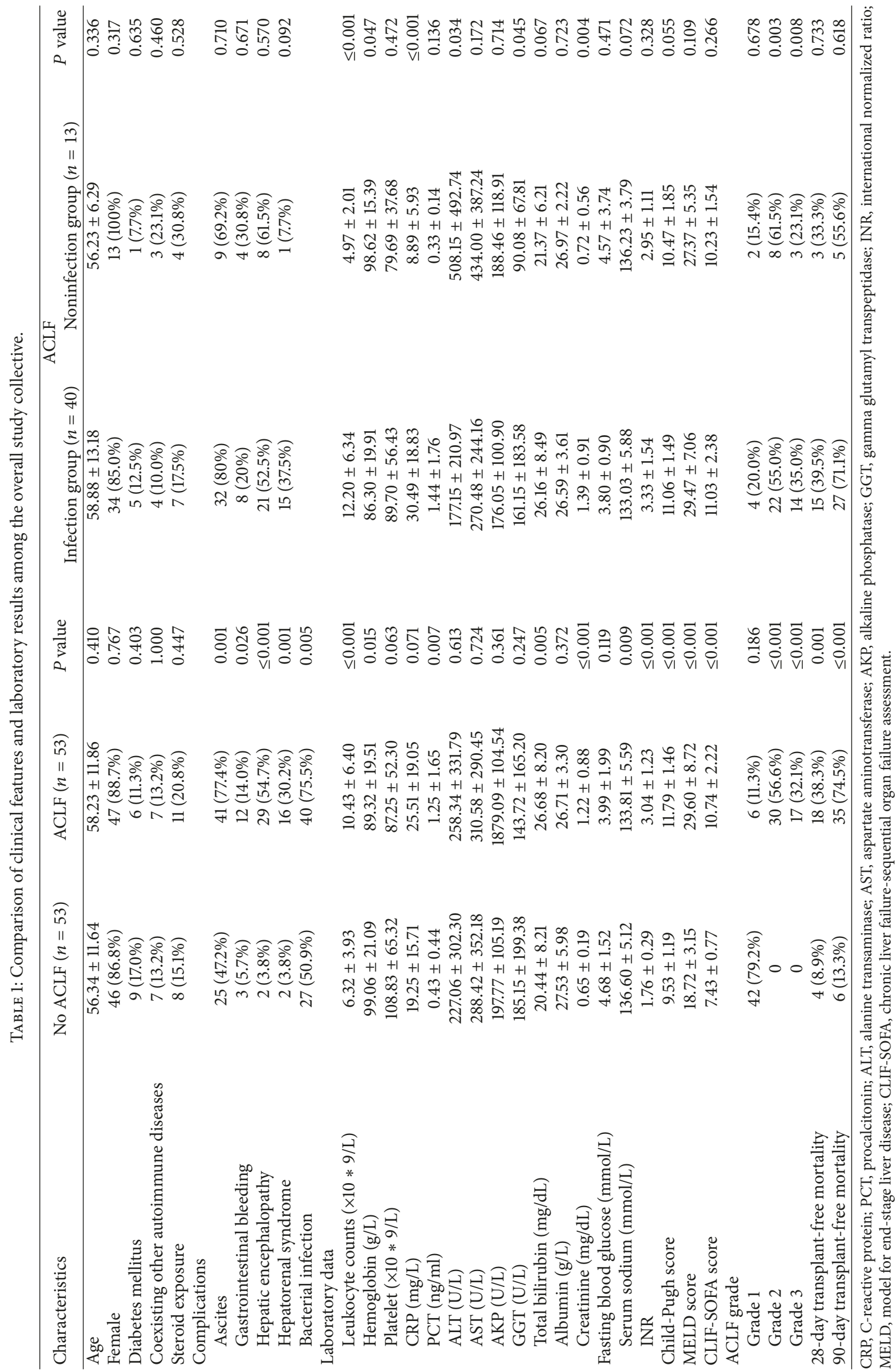


TABLE 2: Significant univariate and multivariate logistic regression analyses of 28-day transplant-free mortality.

\begin{tabular}{|c|c|c|c|c|c|c|}
\hline \multirow{2}{*}{ Variable } & \multicolumn{3}{|c|}{ Univariate } & \multicolumn{3}{|c|}{ Multivariate } \\
\hline & OR & $95 \% \mathrm{CI}$ & $P$ value & OR & $95 \% \mathrm{CI}$ & $P$ value \\
\hline Gastrointestinal bleeding & 5.000 & $1.225-20.409$ & 0.025 & 3.406 & $0.687-16.894$ & 0.134 \\
\hline Hepatic encephalopathy & 3.683 & $1.036-13.100$ & 0.044 & 2.349 & $0.461-11.972$ & 0.304 \\
\hline CLIF-SOFA score & 1.362 & $1.023-1.812$ & 0.034 & 1.093 & $0.741-1.613$ & 0.653 \\
\hline
\end{tabular}

CLIF-SOFA, chronic liver failure-sequential organ failure assessment.

TABLE 3: Significant univariate and multivariate logistic regression analyses of 90 -day transplant-free mortality.

\begin{tabular}{|c|c|c|c|c|c|c|}
\hline \multirow{2}{*}{ Variable } & \multicolumn{3}{|c|}{ Univariate } & \multicolumn{3}{|c|}{ Multivariate } \\
\hline & OR & $95 \%$ CI & $P$ value & OR & $95 \% \mathrm{CI}$ & $P$ value \\
\hline Hepatic encephalopathy & 8.800 & $2.024-38.253$ & 0.004 & 36.714 & $0.085-15810.528$ & 0.244 \\
\hline Leukocyte counts & 1.160 & $1.003-1.342$ & 0.046 & 1.328 & $0.962-1.832$ & 0.085 \\
\hline Hemoglobin & 0.964 & $0.930-1.000$ & 0.048 & 0.912 & $0.808-1.029$ & 0.135 \\
\hline AST & 0.997 & $0.995-1.000$ & 0.021 & 0.998 & $0.992-1.004$ & 0.477 \\
\hline Creatinine & 5.426 & $1.362-21.612$ & 0.016 & 160.487 & $0.049-527959.376$ & 0.219 \\
\hline INR & 2.935 & $1.192-7.224$ & 0.019 & 50.782 & $0.415-6206.850$ & 0.109 \\
\hline MELD score & 1.232 & $1.070-1.418$ & 0.004 & 0.664 & $0.309-1.430$ & 0.295 \\
\hline Child-Pugh score & 2.003 & $1.169-3.430$ & 0.011 & 0.595 & $0.114-2.457$ & 0.473 \\
\hline CLIF-SOFA score & 2.936 & $1.532-5.630$ & 0.001 & 1.578 & $0.350-7.117$ & 0.553 \\
\hline
\end{tabular}

AST, aspartate aminotransferase; INR, international normalized ratio; MELD, model for end stage liver disease; CLIF-SOFA, chronic liver failure-sequential organ failure assessment.

syndrome. The control group included $26(49.1 \%)$ patients of PBC, 24 (45.3\%) patients of $\mathrm{AIH}$, and 3 (5.7\%) cases of PSC. Compared to patients without ACLF, the ACLF group had a higher prevalence of complications $(P<0.05)$, such as hepatic encephalopathy $(54.7 \%$ and $3.8 \%, P \leq 0.001)$ and bacterial infection $(75.5 \%$ and $50.9 \%, P=0.005)$. The 28 day and 90-day mortality rates were also obviously high in patients with $\operatorname{ACLF}(P<0.05)$.

3.2. Risk Factors Associated with 28-Day and 90-Day Mortality. In the study, $6(11.3 \%)$ patients underwent liver transplantation. In the remaining 47 patients, 28 -day mortality and 90-day mortality were $38.3 \%$ (18) and $74.5 \%$ (35), respectively. For the 28-day transplant-free mortality, in univariate analysis, gastrointestinal bleeding $(P=0.025)$, hepatic encephalopathy $(P=0.044)$, and CLIF-SOFA score $(P=0.034)$ were significant factors (Table 2$)$. However, in multivariate analysis, there was no predictor associated with increased mortality. In addition, for the 90-day transplantfree mortality, in univariate analysis, hepatic encephalopathy $(P=0.004)$, leukocyte counts $(P=0.046)$, hemoglobin $(P=0.048)$, aspartate aminotransferase (AST) $(P=0.021)$, serum creatinine $(P=0.016)$, serum sodium $(P=0.017)$, INR $(P=0.019)$, MELD score $(P=0.004)$, Child-Pugh score $(P=0.011)$, and CLIF-SOFA score $(P=0.001)$ were significant factors (Table 3 ). In multivariate logistic regression analysis, no predictor was significantly associated with 90day transplant-free mortality (Table 3).

3.3. Clinical Features of Bacterial Infection in Patients with Autoimmune Liver Disease-Associated ACLF. In our study, $40(75.5 \%)$ patients had bacterial infection, and 14 (35.0\%) patients were diagnosed with bacterial coinfection in the first $72 \mathrm{~h}$ of admission. The demographic and clinical characteristics of ACLF patients with and without bacterial infection are detailed in Table 1.

ACLF patients with bacterial infection showed high Child-Pugh score, MELD score, CLIF-SOFA score, 28- day mortality, and 90-day mortality. Meanwhile, no statistical significance was observed $(P>0.05)$. However, in laboratory findings, bacterial infection patients displayed high leukocyte counts and C-reactive protein (CRP) $(P<0.05)$. High serum levels of serum creatinine and gamma glutamyl transpeptidase (GGT), low levels of hemoglobin, and alanine transaminase (ALT) were also observed in bacterial infection patients $(P<0.05)$ (Table 1$)$.

In 40 ACLF patients with bacterial infection, the most common site of bacterial infection was in the respiratory tract $(17,42.5 \%)$, followed by the peritoneum, bloodstream, biliary tract, urinary tract, intestinal tract, skin and soft tissue, and undetermined site with percentage values of $22.5 \%$ (9), $7.5 \%$ (3), $7.5 \%$ (3), $5.0 \%$ (2), $5.0 \%$ (2), $2.5 \%$ (1), and $7.5 \%$ (3), respectively. The bacteriological evidence of infection was 7 cases $(17.5 \%)$. The pathogens that caused bacterial infections were Escherichia coli in three cases, two of which produced extended spectrum $\beta$ lactamase (ESBL); Klebsiella pneumoniae in two cases; Staphylococcus aureus in two cases, one of which involved methicillin-resistant S. aureus; and Enterococcus faecalis in one case.

3.4. Comparison of CRP and PCT in ACLF Patients with Bacterial Infection. CRP and PCT levels were obtained in 52 and 37 patients, respectively, among 53 patients. Thirty-seven patients had both biomarkers measured simultaneously. 


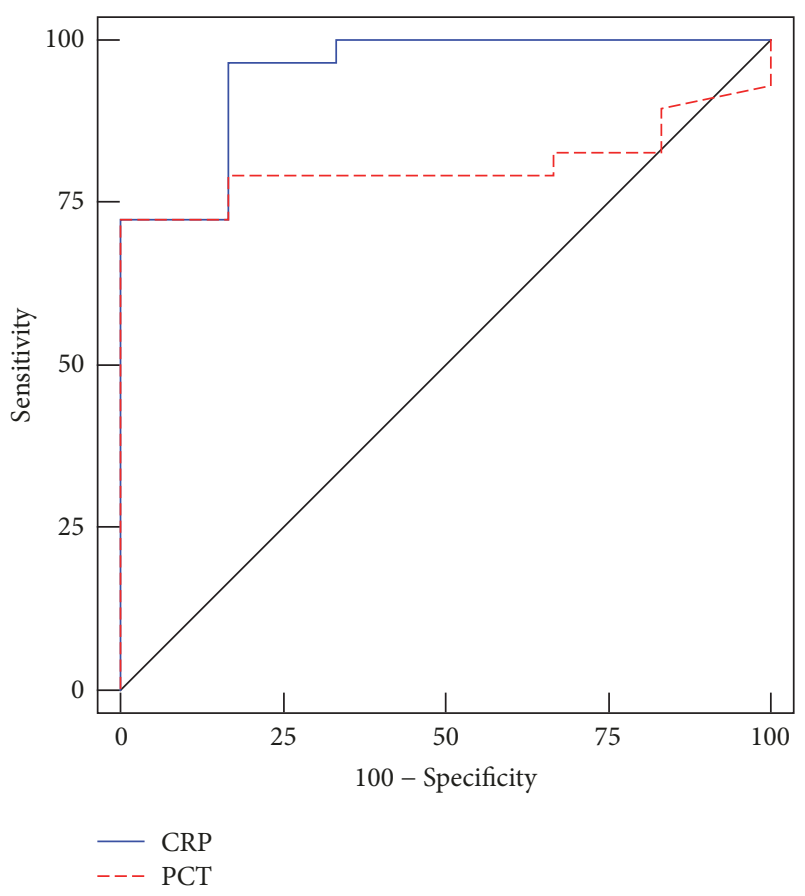

FIGURE 1: Using receiver operating characteristic analysis, comparison of C-reactive protein (CRP) and procalcitonin (PCT) in acuteon-chronic liver failure patients with bacterial infection.

Using receiver operating characteristic analysis, area under curve (AUC) to diagnose bacterial infection was 0.948 (95\% confidence interval $[\mathrm{CI}]: 0-1.000)$ for CRP $(P=0.001)$ compared with 0.807 (95\% CI: 0.668-0.947) for PCT $(P=$ 0.019) (Figure 1). For diagnosis of bacterial infection in CRP at a cut-off value of $12.15 \mathrm{mg} / \mathrm{L}$, the sensitivity and specificity were $96.6 \%$ and $83.3 \%$, respectively. For diagnosis of bacterial infection in PCT at a cut-off value of $0.57 \mathrm{ng} / \mathrm{mL}$, the sensitivity and specificity were $72.4 \%$ and $100 \%$ respectively.

\section{Discussion}

ACLF is a hotspot issues; however, it is lack of uniform diagnostic criteria. Different diagnostic criteria could lead to different patient prognosis. In our study, ACLF patients satisfied the EASL definition, and patients who met APCCMI definition but not EASL definition were included as control group. The 28-day transplantation-free mortality of autoimmune liver disease-associated ACLF was 38.3\%, and 90-day transplantation-free mortality was $74.5 \%$. Of note, a great number of critically ill patients were included in the ACLF group; ACLF grades 2 and 3 (56.6\% and 36.1\%, resp.) were dominant. Moreover, similar to the CANONIC study [4], the ACLF group had a higher prevalence of complications and higher 28-day and 90-day mortality rates.

Several studies were available about predictors of mortality in ACLF patients [5, 14-19]. Yu et al. [14] found that age, etiology, serum sodium, and ascites are independently associated with mortality. Cárdenas et al. [5] considered that the presence of hyponatremia is an independent predictive factor of survival in patients with ACLF. In Mücke's study, infection-triggered ACLF was considered as an independent predictors associated with increased mortality [19]. In our study, no predictor was significantly associated with 28-day and 90-day transplant-free mortality. Small size of study population may cause the situation.

In this study, among the 53 patients with autoimmune liver disease-associated ACLF, 40 (75.5\%) patients had bacterial infection. Bacterial infection in liver disease had been reported by previous documents, and the incidence was 24.4\%-90\% [13, 20-23]. However, study populations were different. Although bacterial infection in ACLF had been studied, autoimmune liver disease-associated ACLF received minimal attention.

In bacterial infection patients with ACLF, Child-Pugh score, MELD score, CLIF-SOFA score, 28-day mortality, and 90-day mortality were high in bacterial infection patients although no statistical significance was observed $(P>0.05)$. ACLF patients with bacterial infection showed a more severe condition than those without infection; this finding was consistent with that of previous study [13].

In recent years, serum CRP and PCT have been suggested for diagnosis and prediction of bacterial infection in chronic liver disease, with or without cirrhosis [13, 24-26]. Papp et al. [25] reported that CRP using a $10 \mathrm{mg} / \mathrm{L}$ cut-off value proved to be more accurate than PCT in identifying patients with infection (AUC: 0.93). In this study, we also revealed that CRP levels are more effective than PCT in the diagnosis of bacterial infection in autoimmune liver disease-associated ACLF. The optimal cut-off value of CRP for bacterial infection diagnosis is $12.15 \mathrm{mg} / \mathrm{L}$ ( $96.6 \%$ sensitivity and $83.3 \%$ specificity), with an AUC of 0.948 , which is similar to previous report [25].

Several limitations were observed in this study. First, this work was a retrospective study at a single center. Some data collections were limited, and bacterial infection might be affected by specific circumstance of our institution. Second, some patients had been treated by antibacterial agents before admission, which might affect the accuracy of bacterial infection rate. Third, immunosuppressor except corticosteroid was not used in our study population. Thus, further studies are needed to investigate bacterial infection and predictors of mortality in autoimmune liver disease-associated ACLF patients using immunosuppressor.

\section{Conclusions}

In conclusion, autoimmune liver disease-associated ACLF displayed high mortality and had more complications. ACLF patients with bacterial infection showed a more severe condition than those without infection. CRP levels higher than $12.15 \mathrm{mg} / \mathrm{L}$ suggested bacterial infection in autoimmune liver disease-associated ACLF patients. No predictor was significantly associated with 28-day and 90-day transplantfree mortality. Further prospective and intervention studies on bacterial infection and predictors of mortality in autoimmune liver disease-associated ACLF patients with large sample numbers are thus needed. 
Abbreviations

$\begin{array}{ll}\text { ACLF: } & \text { Acute-on-chronic liver failure } \\ \text { PBC: } & \text { Primary biliary cholangitis } \\ \text { AIH: } & \text { Autoimmune hepatitis } \\ \text { PSC: } & \text { Primary sclerosing cholangitis } \\ \text { CLIF-SOFA: } & \text { Chronic liver failure-sequential organ } \\ & \text { failure assessment } \\ \text { APPCCMI: } & \text { Asia-Pacific Congress of Clinical } \\ & \text { Microbiology and Infection } \\ \text { EASL: } & \text { European Association for the Study of the } \\ & \text { Liver } \\ \text { CRP: } & \text { C-reactive protein } \\ \text { PCT: } & \text { Procalcitonin } \\ \text { GGT: } & \text { Gamma glutamyl transpeptidase } \\ \text { ALT: } & \text { Alanine transaminase } \\ \text { INR: } & \text { International normalized ratio } \\ \text { MELD: } & \text { End-stage liver disease } \\ \text { AUC: } & \text { Area under curve } \\ \text { CI: } & \text { Confidence interval. }\end{array}$

\section{Conflicts of Interest}

The authors declare that they have no conflicts of interest.

\section{Authors' Contributions}

Xuan Zhang and Ping Chen contribute equally to the study.

\section{Acknowledgments}

This work was supported by the National Science and Technology Major Project (2017ZX10204401002002) and the Zhejiang Provincial Natural Science Foundation (LQ15H030004).

\section{References}

[1] R. Jalan, P. Gines, J. C. Olson et al., "Acute-on chronic liver failure," Journal of Hepatology, vol. 57, no. 6, pp. 1336-1348, 2012.

[2] R. Jalan, C. Yurdaydin, J. S. Bajaj et al., "Toward an improved definition of acute-on-chronic liver failure," Gastroenterology, vol. 147, no. 1, pp. 4-10, 2014.

[3] H. Garg, A. Kumar, V. Garg, P. Sharma, B. C. Sharma, and S. K. Sarin, "Clinical profile and predictors of mortality in patients of acute-on-chronic liver failure," Digestive and Liver Disease, vol. 44, no. 2, pp. 166-171, 2012.

[4] R. Moreau, R. Jalan, P. Gines et al., "Acute-on-chronic liver failure is a distinct syndrome that develops in patients with acute decompensation of cirrhosis," Gastroenterology, vol. 144, no. 7, pp. 1426.e9-1437.e9, 2013.

[5] A. Cárdenas, E. Solà, E. Rodríguez et al., "Hyponatremia influences the outcome of patients with acute-on-chronic liver failure: an analysis of the CANONIC study," Critical Care (London, England), vol. 18, no. 6, p. 700, 2014.

[6] R. Jalan, F. Saliba, M. Pavesi et al., "Development and validation of a prognostic score to predict mortality in patients with acuteon-chronic liver failure," Journal of Hepatology, vol. 61, no. 5, pp. 1038-1047, 2014.
[7] Y. Shi, M. Zheng, and Y. Yang, "Increased delayed mortality in patients with acute-on-chronic liver failure who have prior decompensation," Journal of Gastroenterology Hepatology, vol. 30, no. 4, pp. 712-718, 2015.

[8] L.-J. Li, "13th Asia-Pacific congress of clinical microbiology and infection consensus guidelines for diagnosis and treatment of liver failure," Hepatobiliary \& Pancreatic Diseases International, vol. 12, no. 4, pp. 346-354, 2013.

[9] E. J. Heathcote, "Management of primary biliary cirrhosis. The American Association for the Study of Liver Diseases practice guidelines," Hepatology, vol. 31, no. 4, pp. 1005-1013, 2000.

[10] M. P. Manns, A. J. Czaja, J. D. Gorham et al., "Diagnosis and management of autoimmune hepatitis," Hepatology, vol. 51, no. 6, pp. 2193-2213, 2010.

[11] R. Chapman, J. Fevery, A. Kalloo et al., "Diagnosis and management of primary sclerosing cholangitis," Hepatology, vol. 51, no. 2, pp. 660-678, 2010.

[12] O. Chazouillères, D. Wendum, L. Serfaty, S. Montembault, O. Rosmorduc, and R. Poupon, "Primary biliary cirrhosisautoimmune hepatitis overlap syndrome: clinical features and response to therapy," Hepatology, vol. 28, no. 2, pp. 296-301, 1998.

[13] J. K. Park, C. H. Lee, I. H. Kim et al., "Clinical characteristics and prognostic impact of bacterial infection in hospitalized patients with alcoholic liver disease," Journal of Korean Medical Science, vol. 30, no. 5, pp. 598-605, 2015.

[14] J.-W. Yu, G.-Q. Wang, and S.-C. Li, "Prediction of the prognosis in patients with acute-on-chronic hepatitis using the MELD scoring system," Journal of Gastroenterology and Hepatology, vol. 21, no. 10, pp. 1519-1524, 2006.

[15] S. Alam, B. Lal, V. Sood, and et al., "Pediatric Acute-onchronic Liver Failure in a Specialized Liver Unit: Prevalence, Profile, Outcome and Predictive Factors," Journal of Pediatric Gastroenterology \& Nutrition, vol. 63, no. 4, pp. 400-405, 2016.

[16] C. Dominguez, E. Romero, J. Graciano, J. L. Fernandez, and L. Viola, "Prevalence and risk factors of acute-on-chronic liver failure in a single center from Argentina," World Journal of Hepatology, vol. 8, no. 34, pp. 1529-1534, 2016.

[17] T. Bruns, R. Nuraldeen, M. Mai et al., "Low serum transferrin correlates with acute-on-chronic organ failure and indicates short-term mortality in decompensated cirrhosis," Liver International, vol. 37, no. 2, pp. 232-241, 2017.

[18] K. A. Wlodzimirow, S. Eslami, A. Abu-Hanna, M. Nieuwoudt, and R. A. F. M. Chamuleau, "A systematic review on prognostic indicators of acute on chronic liver failure and their predictive value for mortality," Liver International, vol. 33, no. 1, pp. 40-52, 2013.

[19] M. M. Mücke, T. Rumyantseva, V. T. Mücke et al., "Bacterial infection-triggered acute-on-chronic liver failure is associated with increased mortality," Liver International, 2017.

[20] N. Rolando, F. Harvey, J. Brahm et al., "Prospective study of bacterial infection in acute liver failure: An analysis of fifty patients," Hepatology, vol. 11, no. 1, pp. 49-53, 1990.

[21] N. Rolando, J. Philpott-Howard, and R. Williams, "Bacterial and fungal infection in acute liver failure," Seminars in Liver Disease, vol. 16, no. 4, pp. 389-402, 1996.

[22] A. D. Zider, R. Zopey, R. Garg, X. Wang, T. S. Wang, and J. C. Deng, "Prognostic significance of infections in critically ill adult patients with acute liver injury: a retrospective cohort study," Liver International, vol. 36, no. 8, pp. 1143-1150, 2016. 
[23] R. Moreau and V. Arroyo, "Acute-on-Chronic Liver Failure: A New Clinical Entity," Clinical Gastroenterology and Hepatology, vol. 13, no. 5, pp. 836-841, 2015.

[24] A. Tsiakalos, A. Karatzaferis, P. Ziakas, and G. Hatzis, "Acutephase proteins as indicators of bacterial infection in patients with cirrhosis," Liver International, vol. 29, no. 10, pp. 1538-1542, 2009.

[25] M. Papp, Z. Vitalis, I. Altorjay et al., "Acute phase proteins in the diagnosis and prediction of cirrhosis associated bacterial infections," Liver International, vol. 32, no. 4, pp. 603-611, 2012.

[26] C. Lazzarotto, M. F. Ronsoni, L. Fayad et al., "Acute phase proteins for the diagnosis of bacterial infection and prediction of mortality in acute complications of cirrhosis.," Annals of Hepatology, vol. 12, no. 4, pp. 599-607, 2013. 


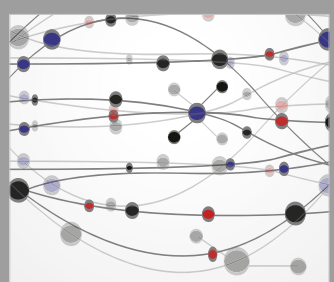

The Scientific World Journal
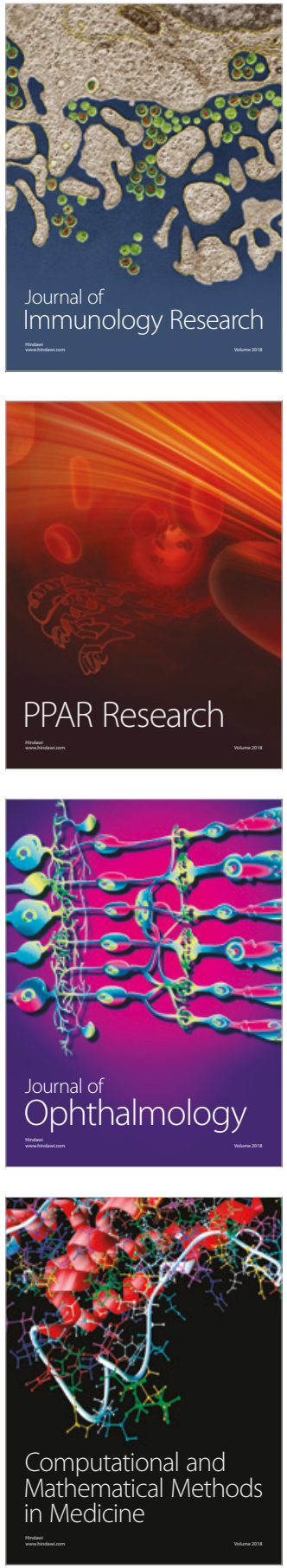

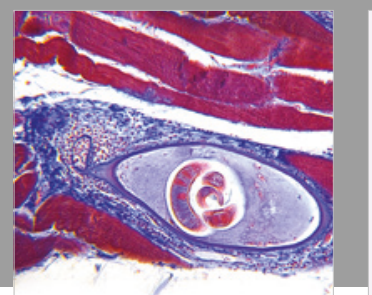

Gastroenterology Research and Practice

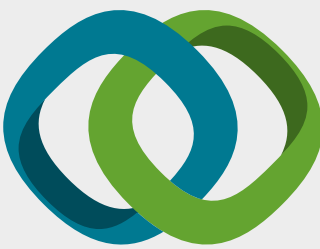

\section{Hindawi}

Submit your manuscripts at

www.hindawi.com
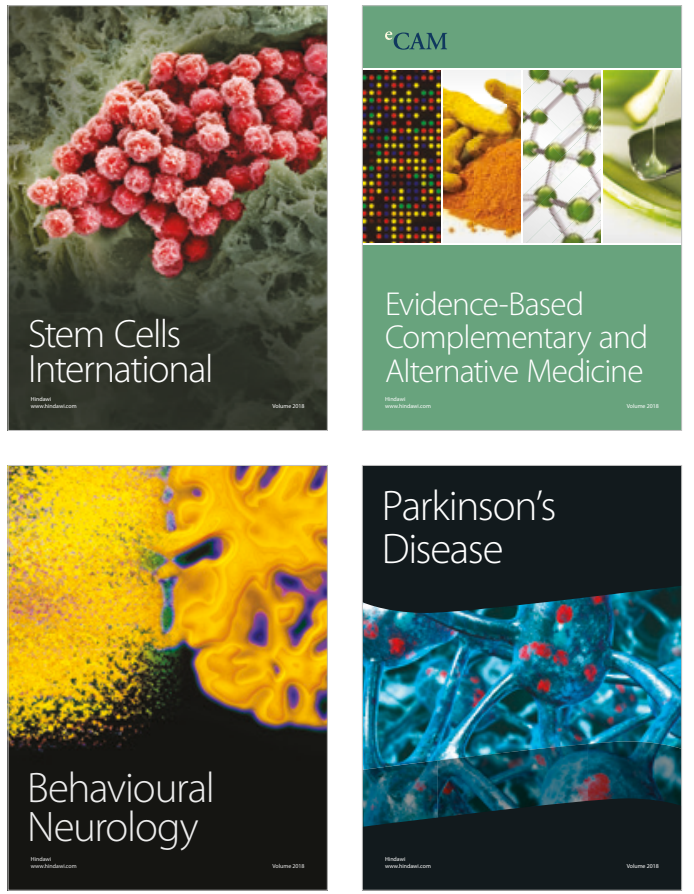

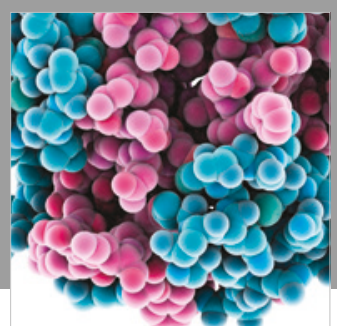

ournal of

Diabetes Research

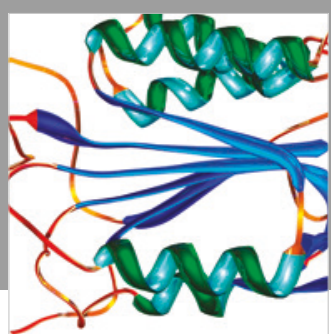

Disease Markers
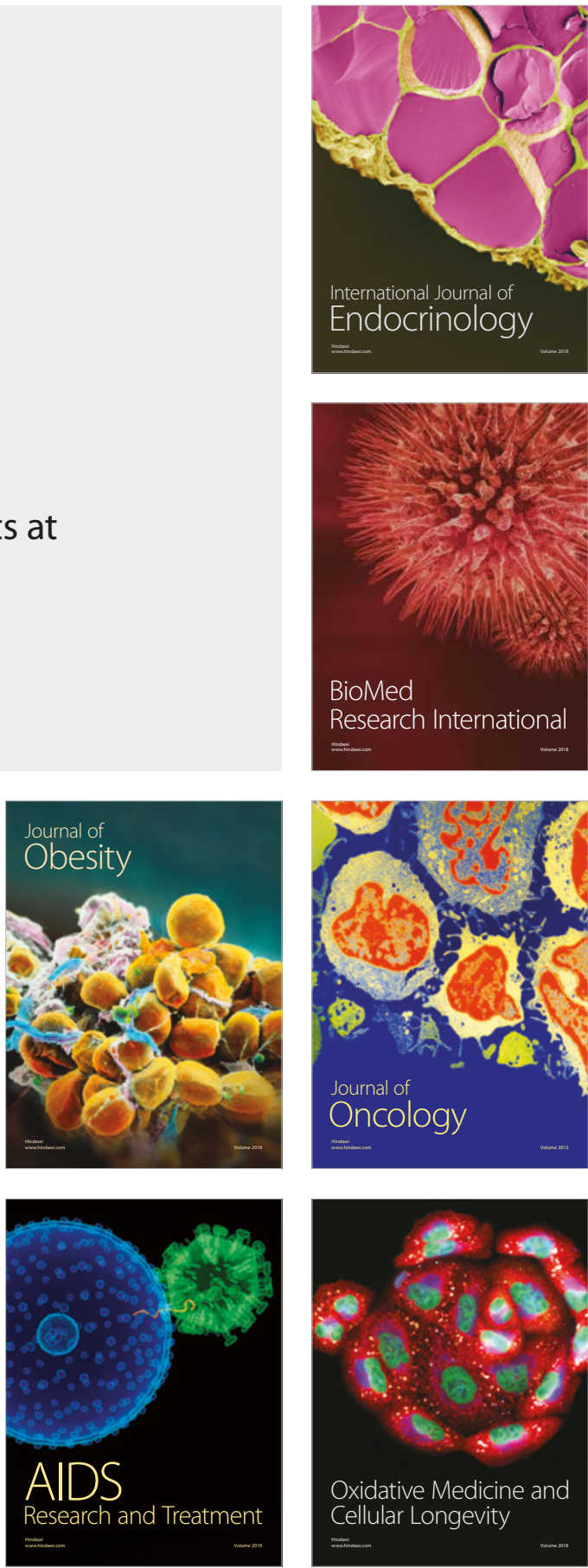